\title{
A PESGA ARTESANAL COM ESPINHEL DE FUNDO NA PLATAFORMA CONTINENTAL AMAZÔNICA
}

\author{
The bottom longline fishery artisanal in the \\ amazon continental shelf \\ Jeanderson da Silva Viana ${ }^{1}$, Rosália Furtado Cutrim Souza² \\ ${ }^{1}$ Mestrando em Aquicultura, bolsista Capes, Centro de Ciências Agrárias (CCA), Universidade Federal de \\ Santa Catarina (UFSC), Rodovia Admar Gonzaga, 1346, Florianópolis - SC, CEP 88034-001 \\ E-mail: jeanderson.viana@posgrad.ufsc.br \\ ${ }^{2}$ Professora adjunta e doutoranda em Ciência Animal na Universidade Federal do Pará (UFPA), \\ Instituto Socioambiental e dos Recursos Hídricos (ISARH), Universidade Federal Rural da Amazônia (UFRA), \\ Avenida Presidente Tancredo Neves, 2501, Belém/PA, CEP 66077-830. E-mail: rosalia.souza@ufra.edu.br
}

\section{RESUMO}

O espinhel é uma arte de pesca passiva que proporciona pouco impacto sobre o fundo oceânico. A pesca com espinhel de fundo na costa amazônica foi caracterizada, no que diz respeito à embarcação utilizada, faina, esforço de pesca e as espécies capturadas. As coletas de dados foram realizadas in loco na embarcação de espinhel da frota artesanal de Bragança-PA, durante 15 lances, distribuídos em três áreas de pesca. A embarcação é considerada de pequeno porte, possui equipamentos necessários para navegação e estruturas específicas para o desenvolvimento das tarefas a bordo. As iscas são obtidas por meio de permuta com embarcações de médio porte. Durante o lançamento e recolhimento de espinhel, 2.600 anzóis são iscados em média por lance. As capturas realizadas na plataforma continental amazônica possuem alta diversidade de peixes, tendo os bagres e os tubarões apresentando os maiores índices de abundância. Mais da metade dos tubarões capturados com espinhel de fundo eram adultos, sugerindo um alto poder de seletividade desse apetrecho e indicando uma potencialidade de uma pesca mais sustentável.

Palavras-chave: pescadores, anzol, isca, bagres, tubarões, pesca sustentável.

\section{ABSTRACT}

The longline is a passive fishing gear and provides little impact on the ocean floor. The bottom longline fishery on the Amazon coast was characterized with respect to the vessel used, work, fishing

Recebido em: 18/08/2018 
effort and species caught. The data collection was carried out in loco on the longline fishing vessels of the artisanal fleet of Bragança-PA for 15 bids, distributed in three fishing areas. The vessel is classified as small size, has the necessary equipment for navigation and specific structures for the development of the tasks on board. The baits are obtained by means of exchange with medium sized vessels. During the launch and gathering of the longline, 2,600 hooks are baited on average per bids. The catches on the amazon continental shelf have high fish diversity, with catfish and sharks showing the highest abundance indexes. More than half of the shark caught in bottom longline was adults, suggesting a high selectivity of this fishing gear indicating a potential for more sustainable fishing.

Keywords: fishermen, fish hook, bait, catfish, sharks, sustainable fishing.

\section{INTRODUÇÃO}

O estuário amazônico é formado pela desembocadura dos rios Tocantins e Amazonas, apresenta uma elevada produtividade biológica e agrega locais de refúgio, reprodução e crescimento de vários peixes (Monteiro et al., 2015). A pesca na região Norte brasileira destaca-se em relação às demais regiões do país por causa da ampla diversidade de espécies explotadas, da quantidade de artes de pesca utilizadas e grande quantidade de pescado capturado, tornando, assim, o eixo principal da exploração de recursos pesqueiros da região amazônica (Oliveira \& Fredóu, 2011; Mendes \& Barthem, 2010).

$\mathrm{Na}$ atividade pesqueira, os pescadores operam com artes de pesca ativas e passivas (Isaac et al., 2008). Entre essas, o espinhel destaca-se por ser um dos métodos que mais satisfaz às premissas da pesca responsável, por permitir a seleção de espécies de grande porte, alta qualidade do pescado, baixo consumo de energia e pouco impacto sobre o fundo oceânico (Mytilineou et al., 2014).

A pesca com linhas e anzóis é dividida em três categorias básicas: linha de mão, pesca de corrico e espinhel, sendo esta última constituída por uma linha principal à qual são conectados os anzóis iscados (Tutuí, 2000). A distância na qual os peixes podem ser atraídos varia principalmente com o tipo de peixe, as iscas utilizadas, o tempo de permanência na água e a direção e intensidade da corrente (Fao, 1976).

O modelo de espinhel de fundo com cabo principal de poliamida começou a ser empregado na região sul para a captura de cações em profundidades de até $300 \mathrm{~m}$ (Peres \& Haimovici, 1998). Na costa norte do Brasil, o espinhel de fundo atua com registros de 242 a 3.000 anzóis que capturam bagres e pescada amarela, espécies alvo, bem como tubarões, considerados fauna acompanhante dessa pescaria, em profundidades de 15 a $70 \mathrm{~m}$ (Alencar et al., 2001; Medeiros \& Santos, 2007). A pesca de tubarões nessa região ocorre em captura direcionada por meio de espinhel e como fauna acompanhante pela frota artesanal e industrial na pesca de bagres marinhos (Morais, 2004).

Dessa forma, pode ser considerada uma arte de pesca adequada para capturar peixes com distribuição dispersa ou com baixa densidade, além de ser possível utilizá-la sobre fundos irregulares ou em grandes profundidades (Bjordal \& Løkkeborg, 1996).

Contudo, há uma escassez de literatura sobre a pesca de espinhel na plataforma continental amazônica e este cenário reflete na gestão inadequada das políticas públicas e na baixa valorização dos pescadores. Com isso, o objetivo do estudo é fornecer um subsídio técnico científico na descrição detalhada da pesca com espinhel de fundo utilizado na plataforma amazônica brasileira, destacando a embarcação utilizada, faina e esforço de pesca. 


\section{MATERIAL E MÉTODOS}

Os registros da atividade pesqueira com espinhel de fundo foram coletados a bordo em outubro de 2016 (estação seca) e janeiro de 2017 (estação de chuva), durante 15 lances, na faixa que abrange a direção da desembocadura da Baía do Marajó (00 $10^{\prime} \mathrm{N} ; 47^{\circ} 92^{\prime} \mathrm{O}$ ) e a costa do município do Oiapoque-AP $\left(03^{\circ} 42^{\prime} \mathrm{N} ; 50^{\circ} 87^{\prime} \mathrm{O}\right)$, entre o estado do Pará e Amapá, entre a isóbata de $25 \mathrm{~m}$ a $35 \mathrm{~m}$ de profundidade (Figura 1).

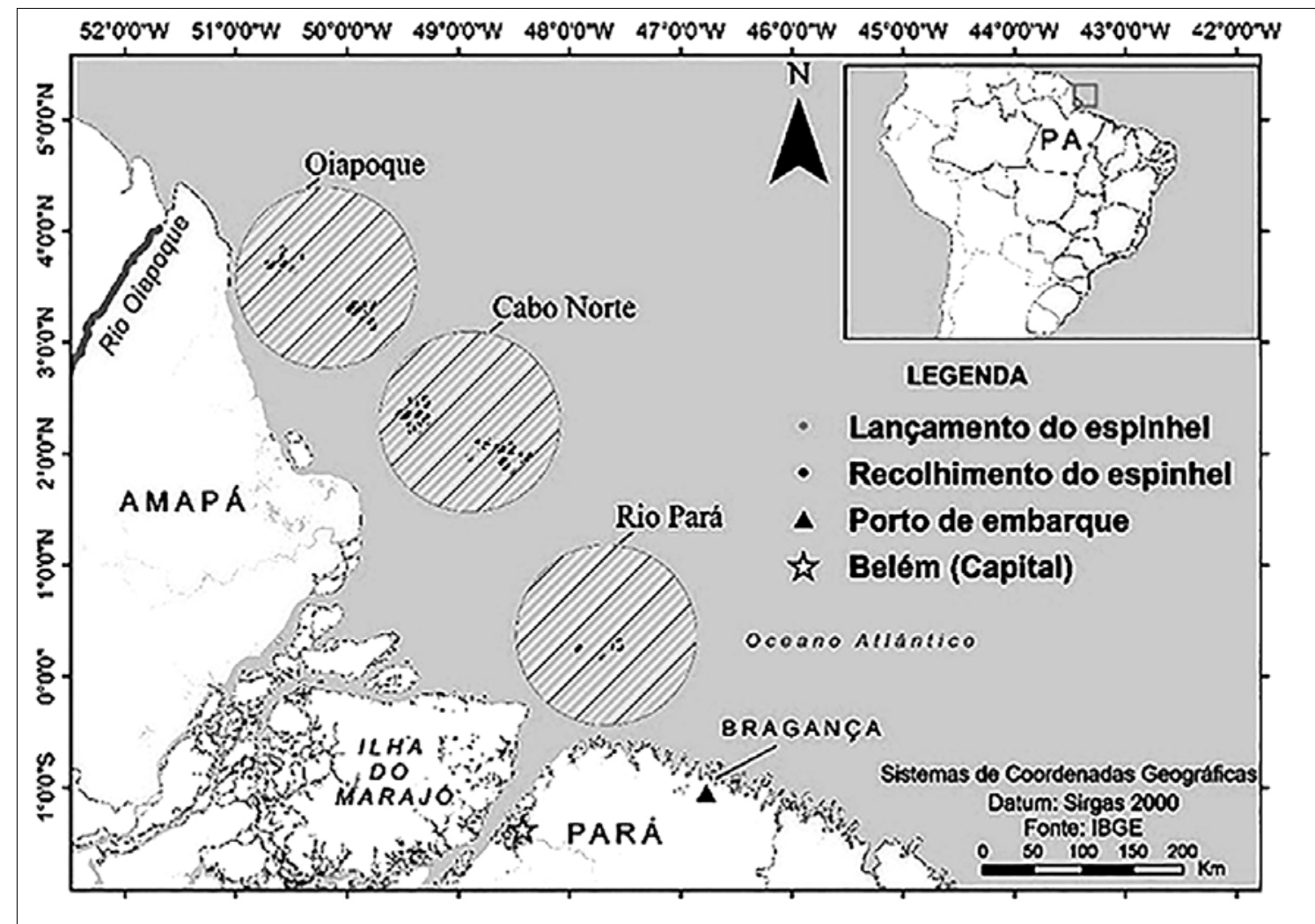

Figura 1 - Locais de captura da pesca de espinhel de fundo na plataforma continental amazônica

A plataforma continental dessa região é a maior do país, com largura média variando entre 133 km (extremo norte da área) e 330 km (frente a foz do rio Amazonas) (Palma, 1979). A pluma superficial de baixa salinidade da plataforma alcança até $270 \mathrm{~km}$ em direção ao mar aberto, devido às águas costeiras oriundas do rio Amazonas (Silva et al., 2005).

As três áreas de atuação da embarcação foram denominadas: "Oiapoque", situada na costa do Amapá; "Cabo Norte”, na costa do estado do Pará; e "rio Pará, situado na desembocadura do rio do mesmo nome, e o porto de origem da embarcação localizou-se no município de Bragança-PA, no nordeste paraense.

No decorrer da viagem, utilizou-se um formulário com o seguinte questionário: material do casco; comprimento total (da extremidade da proa até a extremidade da popa); largura total (mensurado na meia nau) da embarcação; potência e marca do motor utilizado durante a viagem; e o registro de tonelagem bruta. A embarcação foi classificada de acordo com a sua arqueação bruta, conforme a Lei nº 11.959 (Brasil, 2009, art.10):

I - de pequeno porte: quando possui arqueação bruta - AB igual ou menor que 20 (vinte); 
II - de médio porte: quando possui arqueação bruta - $\mathrm{AB}$ maior que 20 (vinte) e menor que 100 (cem);

III - de grande porte: quando possui arqueação bruta - AB igual ou maior que 100 (cem).

Durante as operações de lançamento e recolhimento do espinhel de fundo, a faina de pesca foi avaliada in loco para a coleta dos dados. O anzol e o snap - grampo utilizado para atracar a linha secundária no cabo principal - foram mensurados e categorizados de acordo com Fao (1990) e as espécies capturadas foram identificadas de acordo com Compagno (1984) e Carvalho-Filho (1999).

A abundância dos recursos pesqueiros foi estimada pela CPUE (Captura por Unidade de Esforço). Esse índice foi estimado para cada lance e área de pesca. O esforço de pesca foi calculado em termos de 1.000 anzóis por hora (ah), tendo a captura expressa em kg. Assim, a unidade utilizada para a CPUE foi $\mathrm{kg} / 1.000 \mathrm{ah}$.

\section{RESULTADOS}

A embarcação utilizada na pesca de espinhel de fundo possui 16 metros de comprimento total, 5,20 metros de largura total, arqueação bruta de 17 toneladas, construção em madeira, urna frigorífica com gelo em escama de 25 toneladas, motor a diesel de 6 cilindros e propulsão de $200 \mathrm{HP}$. Em conformidade com sua arqueação bruta (AB), é classificada como embarcação de pequeno porte $(\mathrm{AB}<20 \mathrm{t})$.

A casaria situa-se na popa a meia-nau, sendo dividida em três compartimentos: cozinha, camarotes e cabine. Da meia-nau para a proa, localiza-se o convés parcial, onde ocorre a faina de pesca de recolhimento do espinhel de fundo.

O espinhel de fundo utilizado nas operações de pesca apresenta os seguintes apetrechos: cabo principal multifilamento de $6 \mathrm{~mm}$ de espessura e $17.000 \mathrm{~m}$ de extensão; linha secundária monofilamento de $2 \mathrm{~mm}$ e 1,20 m de comprimento; snap de 0,18 m de comprimento; anzol do tipo torcido $13 / 0$ e garateia de $25 \mathrm{~kg}$. A arte de pesca é composta, em média, por 2.600 linhas secundárias iscadas distantes 1,70 m entre si que são engatadas no cabo principal por meio de snaps. A arte de pesca é fixada sobre o fundo do oceano por meio das garateias e as boias de sinalização são utilizadas para facilitar a sua localização do espinhel de fundo (Figura 2).

As iscas utilizadas nas operações de pesca são obtidas por meio de permuta de alimentos e bebidas com as embarcações de médio porte que operam na plataforma continental amazônica com rede de arrasto. Entretanto, quando essas embarcações não estão operando nas proximidades, são utilizadas redes de emalhe com malha de $9 \mathrm{~cm}$ entre nós opostos para capturar os peixes e utilizá-los como iscas.

As espécies preferidas para serem utilizadas como iscas são os peixes de coloração prateada por permitirem o reflexo da luz solar na água, sendo, consequentemente, mais atrativas para as espécies-alvo: pescada amarela Cynoscion acoupa (Lacèpede, 1802) e tubarões (Sphyrnidae e Carcharhinidae).

Dessa forma, as espécies mais utilizadas como isca são as seguintes: peixe espada Trichiurus lepturus (Linnaeus, 1758), cavala Scomberomons cavalla (Cuvier, 1829), bonito Auxis thazard (Lacepéde, 1800), serra Scomberomorus brasiliensis (Collette; Russo \& ZavallaCamin, 1978) e pescada-gó Macrodon ancylodon (Bloch \& Schneider, 1801). Todas são conservadas em gelo, porém, esta última diferencia-se das demais, pois, antes da sua conservação, aplica-se o processamento de salga. 


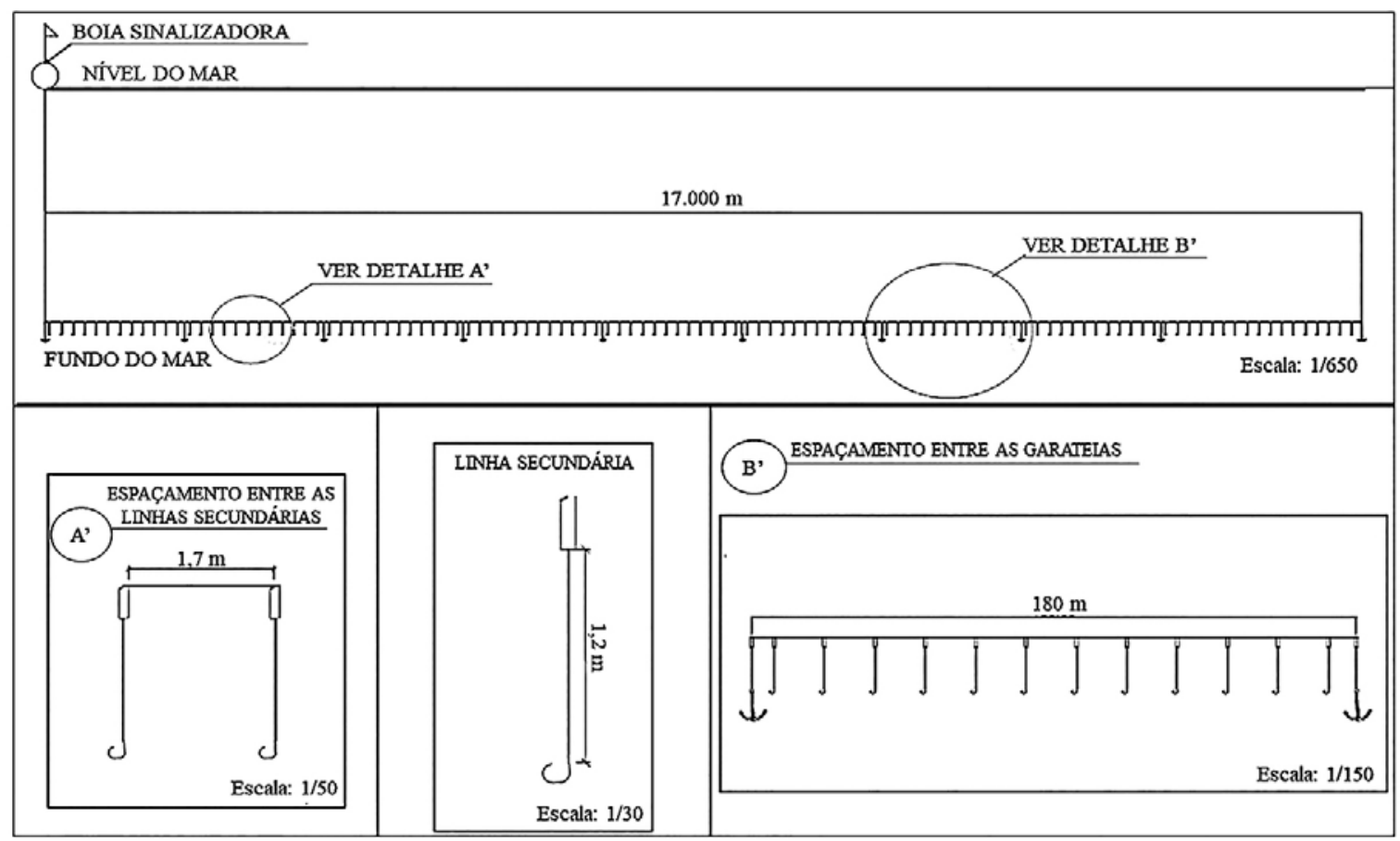

Figura 2 - Representação do espinhel de fundo utilizado nas pescarias realizadas na plataforma continental amazônica

Antes da operação de pesca, os pescadores realizam o iscamento dos anzóis no convés da embarcação e agrupam as linhas secundárias iscadas em lotes de 50 unidades para facilitar na contagem dos anzóis lançados e na praticidade da faina de pesca.

No início do lançamento do espinhel, os pescadores atracam uma âncora na extremidade do cabo principal e lança-o no mar pela popa da embarcação a uma velocidade de 6 a 7 nós, geralmente na mesma direção da corrente. Em seguida, um pescador engata as linhas secundárias ao longo do cabo principal numa distância de 1,7 m entre si, e também atraca as âncoras de $25 \mathrm{~kg}$ a cada $180 \mathrm{~m}$, que são utilizadas para fixar o material sobre o fundo do oceano. Ao término da operação, uma boia de sinalização é atrelada no cabo principal para facilitar o mestre de pesca em relação à localização do espinhel de fundo. Em média, a faina do lançamento de espinhel dura 3 horas.

No recolhimento do material, um dos pescadores recolhe o cabo principal por meio da garateia e atraca-o no guincho elétrico para retirar o material da água. Durante a operação, um pescador desatraca as linhas secundárias do cabo principal e lança-as no convés da embarcação. Simultaneamente, os pescadores realizam a limpeza do anzol removido da boca do peixe, evisceram os indivíduos capturados e armazenam na urna com gelo em escama. A operação de recolhimento dura em torno de 2 horas. A tabela I apresenta as principais características dos 15 lançamentos de espinhel de fundo na plataforma amazônica.

Tabela I - Produção e esforço de pesca dos lançamentos realizados pela embarcação da pesca de espinhel de fundo na plataforma continental amazônica

\begin{tabular}{lcccc}
\hline Variáveis & Valor mínimo & Valor médio & Valor máximo & Desvio padrão \\
\hline Captura total $(\mathrm{kg})$ & 51,7 & 288,5 & 814,5 & 223,5 \\
$\mathrm{~N}^{\circ}$ de indivíduos capturados & 19 & 110,27 & 254 & 75,72 \\
Tempo de imersão/lance $(\mathrm{h})$ & 04 & $05: 49$ & $09: 13$ & $01: 51$ \\
$\mathrm{~N}^{\circ}$ de anzóis & 2100 & 2603,3 & 3000 & 294,27 \\
Esforço $(1000 \mathrm{a} \times \mathrm{h})$ & 8,74 & 14,97 & 22,74 & 4,43 \\
CPUE $(\mathrm{kg} / 1000 \mathrm{a} \times \mathrm{h})$ & 3,15 & 19,60 & 55,91 & 14,11
\end{tabular}


No total, foram capturados 1.656 peixes, pertencentes a duas classes (Actinopterygii e Elasmobranchii), sete ordens, 14 famílias, 20 gêneros e 24 espécies. Em relação à CPUE, a uritinga foi a espécie mais abundante $(6,87 \mathrm{~kg} / 1.000$ anzol-hora) durante a viagem, sendo que a presença dos peixes da família Ariidae mostra-se relevante na produção pesqueira. No que se refere aos tubarões da família Carcharhinidae, o cação frango foi mais abundante (Tabela II).

\begin{tabular}{|c|c|c|c|}
\hline Família & Nome científico & Nome vulgar & Índice de abundância \\
\hline Ariidae & Sciades proops & Uritinga & 6,87 \\
\hline Carcharhinidae & Rhizoprionodon porosus & Cação frango & 4,40 \\
\hline Ariidae & Sciades parkeri & Gurijuba & 4,24 \\
\hline Ariidae & Aspistor quadriscutis & Cangatan & 2,91 \\
\hline Ariidae & Bagre bagre & Bandeirado & 2,76 \\
\hline Sciaenidae & Cynoscion acoupa & Pescada amarela & 2,70 \\
\hline Ginglymostomatidae & Ginglymostoma cirratum & Tubarão lixa & 2,53 \\
\hline Carcharhinidae & carcharhinus porosus & Cação prenhoca & 2,21 \\
\hline Sciaenidae & Cynoscion virescens & Corvina & 2,11 \\
\hline Sphyrnidae & Sphyrna tudes & Tubarão martelo & 2,06 \\
\hline Dasyatidae & Dasyatis guttata & Arraia bicuda & 2,04 \\
\hline Ariidae & Hemiarius grandicassis & Cambéua & 2,02 \\
\hline Pomatomidae & Pomatomus saltatrix & Anchova & 1,32 \\
\hline Carcharhinidae & Galeocerdo cuvier & Jaguara & 1,22 \\
\hline Carcharhinidae & Carcharhinus limbatus & Sacuri galha preta & 0,63 \\
\hline Carangidae & Caranx hippos & Xaréu & 0,58 \\
\hline Rachycentridae & Rachycentron canadum & Beijupirá & 0,38 \\
\hline Echeneidae & Remora brachyptera & Rêmora & 0,32 \\
\hline Carcharhinidae & Carcharhinus leucas & Cação bebéu & 0,23 \\
\hline Batrachoididae & Batrachoides surinamensis & Pacamom & 0,21 \\
\hline Tetraodontidae & Lagocephalus laevigatus & Baiacu bandeira & 0,13 \\
\hline Lutjanidae & Lutjanus synagris & Ariacó & 0,10 \\
\hline Echeneidae & Gymnothorax sp. & Moreia & 0,06 \\
\hline Triakidae & Mustelus canis & Boca de velha & 0,04 \\
\hline
\end{tabular}

A maior captura ocorreu na área de pesca "Oiapoque" durante o lance 1, tendo como destaque 118 tubarões capturados, entre eles: tubarão-martelo (Sphyrna tudes) (Valenciennes, 1822), cação prenhoca (Carcharhinus porosus) (Ranzani, 1839), cação frango (Rhizoprionodon porosus) (Poey, 1861), sacuri galha preta (Carcharhinus limbatus) (Müller \& Henle, 1839), e cação bebéu (Carcharhinus leucas) (Müller \& Henle, 1839).

A experiência de anos anteriores do patrão de pesca na pesca artesanal pode refletir na escolha das áreas de pesca. No "Oiapoque", o espinhel permaneceu no fundo do oceano, aproximadamente por $15 \mathrm{~h}$, devido à pane ocorrida no guincho elétrico utilizado para recolher o cabo principal durante o recolhimento do espinhel. Com o espinhel de fundo inerte no fundo do mar, os tubarões romperam o cabo e se alimentaram dos peixes capturados pelos anzóis. Após o conserto do guincho, o cabo retornou a ser recolhido, mesmo com a produção afetada.

Entre as capturas por unidade de esforço realizadas na plataforma continental amazônica, o maior índice de abundância ocorreu em um único lance realizado, nas proximidades da foz do rio Pará $00^{\circ} 15^{\prime} \mathrm{N}$ (Figura 3). 


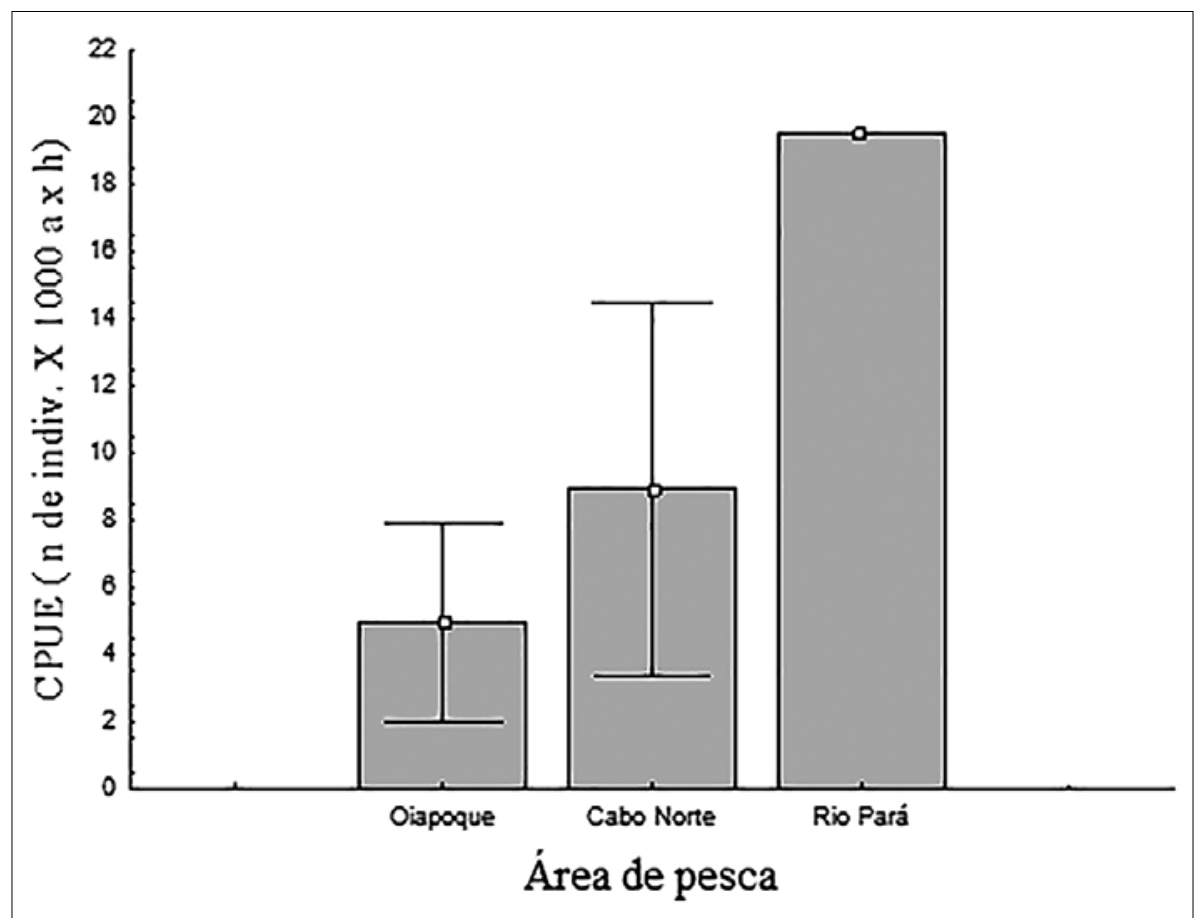

Figura 3 - Índice de abundância média (CPUE) de cada área de pesca com espinhel de fundo na plataforma continental amazônica e seus respectivos desvios padrões

Ao longo da viagem, ocorre um decréscimo no esforço de pesca, na costa do Amapá, uma vez que o número de anzóis é reduzido em relação ao primeiro lançamento por causa da escassez de iscas. Esse fato reflete na diminuição da produção da pescaria.

Na área de pesca "Cabo Norte", os lançamentos são suspensos após os lances 10 e 11 demonstrarem baixa produtividade devido ao esforço de pesca elevado nos lances superiores. Em busca de áreas oportunas de pesca, a embarcação segue em direção às águas barrentas da área pesqueira "Rio Pará", onde realiza o lance 13 e obtém o maior índice de abundância.

Por causa das grandes capturas de bandeirado Bagre bagre (Linnaeus, 1766), os pescadores utilizam essa espécie como iscas nos lances seguintes. Dessa forma, os últimos lançamentos são favorecidos por apresentarem o número máximo de anzóis iscados.

As áreas de pesca mais explotadas situam-se acima de $2^{\circ} 00^{\prime} \mathrm{N}$, uma vez que a área do Oiapoque e do Cabo Norte abrangeram 73,3\% dos lançamentos de espinhel de fundo, sendo, assim, as áreas mais atraentes aos pescadores dessa arte de pesca, apesar da dificuldade em encontrar outra embarcação de pesca do campo de vista do patrão de pesca.

No total, foram capturados 1.654 indivíduos com biomassa total de $4.327 \mathrm{~kg}$, abrangendo 25 espécies: 88,9\% Actinopterygii e 11,1\% Elasmobranchii, sendo que as famílias Carcharhinidae e Ariidae foram as mais dominantes na amostragem.

A família Carcharhinidae foi representada pelas seguintes espécies: cação prenhoca, cação frango, sacuri galha preta e cação bebéu. Em relação à família Ariidae, os seguintes bagres foram capturados: uritinga Sciades proops (Valenciennes, 1840), gurijuba Sciades parkeri (Traill, 1832), cambéua Hemiarius grandicassis (Valenciennes in Cuvier; Valenciennes, 1840), cangatá Aspistor quadriscutis (Valenciennes, 1840) e bandeirado Bagre bagre (Linnaeus, 1766).

A pescada amarela foi o peixe mais almejado durante a pescaria, por possuir um importante valor econômico por causa da comercialização da sua bexiga natatória, deno- 
minada comumente de "grude". Em relação aos tubarões, os pescadores não realizam a prática do finning devido a uma forte fiscalização dos órgãos competentes e pelas nadadeiras não possuírem valor econômico tão expressivo em relações a tempos anteriores.

Em relação aos locais de captura, na área de pesca “Oiapoque" foram capturados 681 indivíduos pesando no total $1.997 \mathrm{~kg}$, onde se observou a predominância de bandeirado $B$. bagre, cambéua H. grandicassis, pescada amarela C. acoupa e uritinga S. proops. O cação prenhoca C. porosus foi o mais representativo entre os elasmobrânquios (Figura 4).

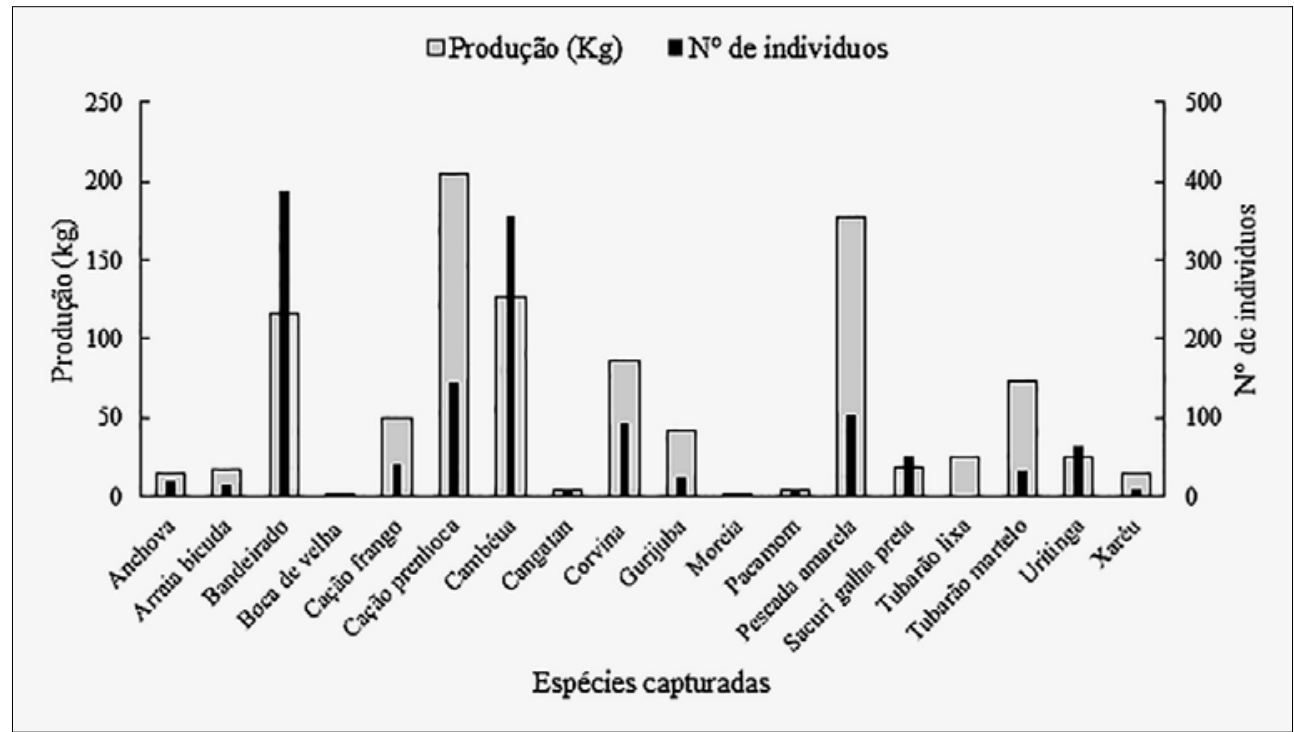

Figura 4 - Produção e número dos indivíduos capturados pela embarcação de espinhel de fundo na área de pesca "Oiapoque"

Na área de pesca "Cabo Norte", foram capturados 719 indivíduos com uma produção total de $1.603 \mathrm{~kg}$. As espécies bandeirado, gurijuba, uritinga, cangatá e corvina merecem destaque no que se refere à produção capturada (Figura 5).

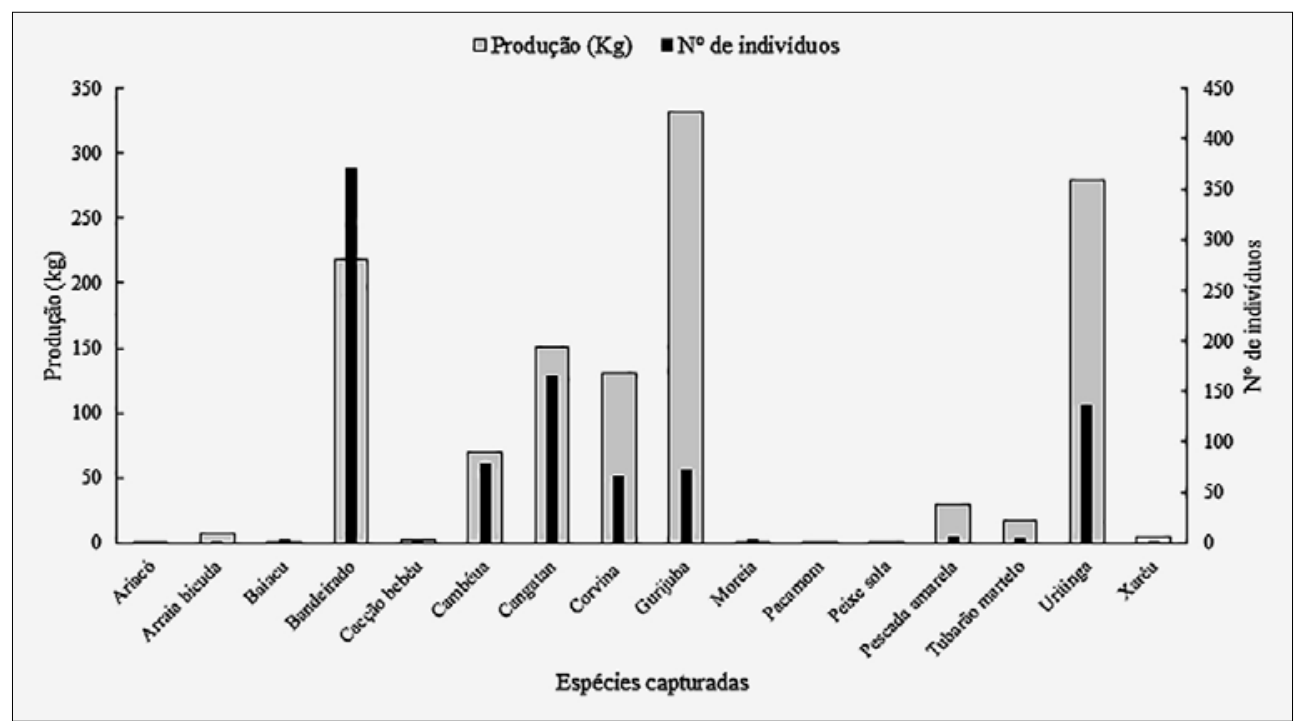

Figura 5 - Produção e número dos indivíduos capturados pela embarcação de espinhel de fundo na área de pesca "Cabo Norte" 
Na área de pesca "rio Pará", foram capturados 254 indivíduos com uma produção total de $726.8 \mathrm{~kg}$, onde observou-se predominância do bandeirado, gurijuba, uritinga, cangatá e corvina (Figura 6).

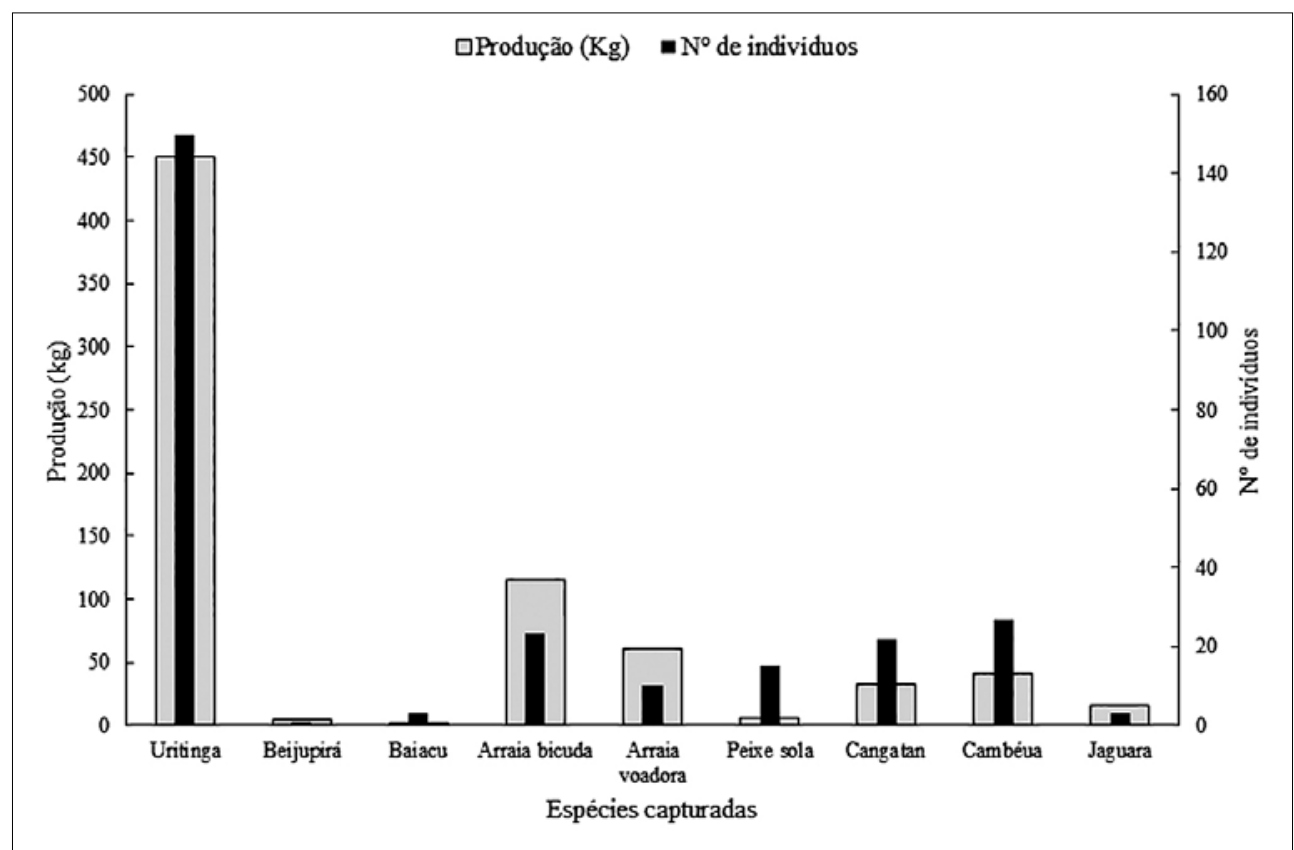

Figura 6 - Produção e número dos indivíduos capturados pela embarcação de espinhel de fundo na área de pesca "rio Pará"

\section{DISCUSSÃO}

A embarcação utilizada na plataforma continental amazônica e os seus apetrechos de pesca apresentam características similares às embarcações que operam na região Sul e Sudeste brasileiro (Haimovici et al., 2004). A maioria das embarcações de espinhel de fundo possuem semelhanças em relação ao lançamento do cabo principal em navegação constante, à presença de equipamentos de navegação, de guinchos elétricos e de iscas (Ávila da Silva; Bastos \& Tutuí, 2001; Haimovici et al., 2004; Nogueira, 2015; Lira, 2016).

Entretanto, nessas regiões do Brasil, as iscas são adquiridas nos portos. Além de utilizarem peixes de baixo valor comercial, os pescadores manuseiam a lula argentina Illex argentinus (Ávila da Silva; Bastos \& Tutuí, 2001; Haimovici et al., 2004). Conforme o conhecimento empírico da tripulação, a escolha da isca está vinculada com a espécie-alvo da pescaria, a coloração da água e as condições de mar da área de pesca. A elevada dependência da aquisição de iscas adia o início da operação de lançamento do espinhel e, consequentemente, resulta na diminuição da produtividade da pesca.

Em relação à arte de pesca, houve diferenças em relação ao tamanho do apetrecho, em que na região Sudeste constitui-se basicamente de um cabo principal de aço de 6 a 7 milhas náuticas de comprimento com cabos secundários de náilon com cerca de $90 \mathrm{~cm}$ de comprimento e 1,8 a 2,0 mm de diâmetro. As embarcações arrendadas da frota japonesa que atuam no Nordeste brasileiro possuem um sistema de espinhel com cerca de $150 \mathrm{~km}$ de cabo principal e 3.000 anzóis, tendo em vista que essas embarcações apresentam $49 \mathrm{~m}$ de comprimento e 400 t de arqueação bruta (Araújo et al., 2013). As características da em- 
barcação de espinhel da região norte são semelhantes às dos apetrechos de pesca utilizados no N/Pq Orion, descritas por Tiago et al. (1995), quando essa pescaria foi introduzida, não havendo modificações (Cepsul, 1998; Wahrlich, 2004; Haimovici et al., 2005).

Durante a viagem, o tempo de imersão do espinhel de fundo foi influenciado pela direção do vento, coloração da água e pelas condições de mar calmo nos locais de captura. Segundo o patrão de pesca, responsável pela viagem, as condições de mar calmo, navegação com vento a favor da embarcação e a coloração verde da água resultam em um ótimo rendimento pesqueiro.

A predominância de peixes de grande porte está associada com a seletividade do anzol, onde peixes pequenos não podem ser capturados por grandes anzóis, enquanto os maiores não são fisgados por anzóis pequenos (Sparre \& Venema, 1992; Meneses et al., 2011; Ingólfsson et al. 2017). Esse tipo de apetrecho foi eficiente na captura de tubarões adultos em 53,3\% dos lances realizados, sendo um resultado positivo, mas que ainda pode ser melhorado, uma vez que esses animais possuem uma baixa fecundidade, maturidade sexual tardia e elevada longevidade, o que os torna bem mais suscetíveis à sobrepesca que os peixes teleósteos (Stevens et al., 2000; Gulak et al., 2015; Yulianto et al., 2018).

Os resultados obtidos em relação à produção confirmam a dominância de bandeirado, gurijuba, uritinga, tubarão e pescada amarela na costa Norte, conforme previamente apresentado no estudo de Lima (2011). A pesca com espinhel de fundo é direcionada para peixes bentônicos, porém também são capturadas espécies demersais que habitam próximo ao substrato, o que reflete uma alta diversidade ictiológica na produção pesqueira.

Nas áreas de pesca próximas da desembocadura do rio Pará, há uma grande quantidade de material orgânico em suspensão e um elevado aporte de nutrientes que contribui para a elevada produtividade pesqueira (Siqueira; Pereira \& Aprile, 2006; Furtado, 2002). Esse fato favorece a alta diversidade da ictiofauna e, consequentemente, pode influenciar nos valores de abundância, gerando pescarias produtivas por meio da captura com espinhel de fundo.

\section{CONCLUSÃO}

As embarcações que utilizam o espinhel de fundo na costa amazônica apresentam um baixo poder de operacionalização, tanto na sua infraestrutura quanto no seu planejamento da faina pesqueira a bordo, com isso, a atividade pode ser caracterizada como artesanal. As capturas realizadas na plataforma continental amazônica possuem alta diversidade de peixes e as espécies mais abundantes são os bagres e os tubarões. Mais da metade dos tubarões capturados com espinhel de fundo eram adultos, sugerindo um alto poder de seletividade desse apetrecho e indicando uma potencialidade de uma pesca menos predatória.

\section{REFERÊNCIAS BIBLIOGRÁFICAS}

Araújo, P.V.D.N.; Ruivo, U.; da Silva, G. B.; Freire, J. \& Bezerra, M. Descrições gerais da frota japonesa arrendada para a pesca de atuns e afins na zona econômica exclusiva do Brasil. Arquivos de Ciências do Mar, v. 46, n. 2, p. 55-63, 2013.

Alencar, C.A.G.; Santana, J.V.M. \& Oliveira, G.G. Descrição da pesca de tubarões com espinhel de fundo na região Norte do Brasil, durante 1996 e 1997. Arquivos de Ciências do Mar, v. 34, n. 1-2, p. 143-149, 2001. 
Ávila-da-Silva, A.O.; Bastos, G.C.C. \& Tutui, S.L.S. A atividade pesqueira do estado de São Paulo: análise das capturas do biênio 1998-1999 com espinhel-de-fundo. Boletim do Instituto de Pesca, v. 27, n. 1, p. 33-38, 2001.

Bjordal, A. \& Løkkeborg, S. Longlining. Fishing News Books, 156 p., Oxford, 1996.

Brasil. Lei $\mathrm{n}^{\mathrm{o}}$ 11.959, de 29 de junho de 2009. Dispõe sobre a Política Nacional de Desenvolvimento Sustentável da Aquicultura e da Pesca. Diário Oficial da União, Brasília, DF, v. 1, 30 jun. 2009. Seção II, p. 5.

Carvalho-Filho, A. Peixes: costa brasileira. Edição Marca D’água, São Paulo, 320 p. 1999.

Cepsul. Centro Nacional de Pesquisa e Conservação da Biodiversidade Marinha do Sudeste e Sul. Peixes demersais: relatório da $4^{\mathrm{a}}$ REUNIÃO DO GRUPO PERMANENTE DE ESTUDOS, realizada no período de 8 a 12 novembro de 1993. Itajaí. Coleção Meio Ambiente. Série Estudos Pesca, n. 16, 1995, 127 p.

Compagno, L.J.V. Sharks of the world. An annotated and illustrated catalogue of shark species known to date, Part 2, Carcharhiniformes. FAO sp. cat., v. 4, n. 2, p. 251-655, 1984.

Cunha, I.B.S.; Sawaya, A.C.H.F.I.; Caetano, F.M.; Shimizu, M.T.; Marcucci, M.C.; Drezza, F.T.; Povia, I.G.S. \& Carvalho, P.O. Factors that influence the yield and composition of Brazilian propolis extracts. J. Braz. Chem. Soc. 2004, v. 15, n. 6, p. 964-970, 2004.

Fao. Food and Agriculture Organization of the United Nations. Monitoring on fish stock abundance: The use of catch and effort data. FAO Fisheries Technical Paper, Rome, 101 p., 1976.

Fao. Food and Agriculture Organization of the United Nations. Guia Prático do Pescador. EDITAMAR, Edições Marítimas. Lisboa,195 p., 1990.

Furtado, L.G. Pesqueiros reais e pontos de pesca: traços da territorialidade haliêutica ou pesqueira amazônica. Boletim do Museu Paraense Emílio Goeldi, v. 18, n. 1, 24 p., 2002.

Gulak, S.J.B.; de Ron Santiago, A.J. \& Carlson, J.K. Hooking mortality of scalloped hammerhead Sphyrna lewini and great hammerhead Sphyrna mokarran sharks caught on bottom longlines. African Journal of Marine Science, v. 37, n. 2, p. 267-273, 2015.

Haimovici, M.; Ávila-da-Silva; A.O.; Tutuí, S.L.L.; Bastos, G.C.C; Santos, R.A. \& Fischer, 1.G. Prospecção pesqueira de espécies demersais com espinhel-de-fundo na região Sudeste-Sul do Brasil, Série Relatórios REVIZEE-Score Sul Inst. Ocean., p. 11-78, 2004.

Ingólfsson, Ó.A.; Einarsson, H.A. \& Løkkeborg, S. The effects of hook and bait sizes on size selectivity and capture efficiency in Icelandic longline fisheries. Fisheries Research, v. 191, n.1, p. 10-16, 2017.

Isaac, V.J.I.; Roberto, V.; Espírito Santo, S. \& José, 1.N.A. Estatística pesqueira no litoral do Pará: resultados divergentes. Panam J. Aquat. Sci., v. 3, n. 3, p. 205-213, 2008.

Lima, V.A.V. Embarcações e artes de pesca utilizadas nos municípios de Calçoene e Oiapoque, no estado do Amapá, Brasil. Monografia, curso de Engenharia de Pesca, Universidade do Estado do Amapá, 51 p., Amapá, 2011.

Lira, M.G. Pesca de atuns e afins no Oceano Atlântico: interações oceanográficas, implicações socioeconômicas e tecnológicas. Dissertação de Mestrado, Programa Regional de PósGraduação em Desenvolvimento e Meio Ambiente, Universidade Federal do Rio Grande do Norte, 80 p., Natal, 2016. 
Medeiros, R.S \& Santos, J.O. Relatório anual da pesca no estado do Amapá. Estatpesca 2006 e parcial de 2007, Macapá, p. 3-44, 2007.

Mendes, F.L.S. \& Barthem, R.B. Hábitos alimentares de bagres marinhos (Siluriformes: ariidae) do estuário amazônico. Amazônia: Ci. E Desenv., v. 5, n. 10, 2010.

Meneses, T.S.; Pereira, C.W. \& Santos, F.N. Pequenos tubarões costeiros capturados por espinhel de fundo operado por embarcação artesanal no litoral de Sergipe. Arquivos de Ciências do Mar, v. 44, n. 1, 2011.

Morais, L.C. Biologia de elasmobrânquios da costa Norte do Brasil. Monografia de graduação, Oceanografia, Universidade Federal do Pará, 57 p., Belém, 2004.

Monteiro, S.M.; El-Robrini, M. \& Alves, I.C.C. Dinâmica sazonal de nutrientes em estuário amazônico. Mercator, v. 14, n. 1, p. 151-162, 2015.

Mytilineou, C.; Smith, C.J.; Anastasopoulou, A.; Papadopoulou, K.N.; Christidis, G.; Bekas, P. \& Dokos, J. New cold-water coral occurrences in the Eastern Ionian Sea: Results from experimental long line fishing. Deep Sea Res. Part II: Top. Stud. in Ocean., v. 99, n. 1, p. 146157, 2014.

Nogueira, M.M. A pesca artesanal e as tartarugas marinhas no litoral paraibano: aspectos etnozoológicos e conservacionistas. Dissertação de mestrado, Programa Regional de PósGraduação em Desenvolvimento e Meio Ambiente, Universidade Federal da Paraíba, 74 p., 2015.

Oliveira, D.M. \& Frédou, F.L. Caracterização e dinâmica espaçotemporal da atividade pesqueira na Baía de Marajó - Estuário Amazônico. Arquivos de Ciências do Mar, v. 44, n. 3, p. 40-53, 2011.

Palma, J.J.C. Geomorfologia da plataforma continental norte brasileira. Série Projeto Remac, v. 7, n. 1, p. 25-51, 1979.

Peres, M.B. \& Haimovici, M. A pesca dirigida ao cherne - poveiro Polyprion americans no sul do Brasil. Atlântico, v. 20, n. 1, p. 141-161, 1998.

Silva, A.C.; Araújo, M. \& Bourles, B. Variação sazonal da estrutura de massas de água na plataforma continental do Amazonas e área oceânica adjacente. Rev. Bras. Geof. São Paulo, v. 23, n. 2, p. 145-157, 2005.

Siqueira, G.W.; Pereira, S.F.P. \& Aprile, F.M. Determinação dos elementos-traço (Zn, Co e $\mathrm{Ni}$ ) em sedimentos da Plataforma Continental Amazônica sob influência da descarga do rio Amazonas. Acta Amaz., v. 36, n. 1, p. 321-326, 2006.

Sparre, P. \& Venema, S.C. Introduction to tropical fish stock assessment. FAO Fish. Tec. Pap., Rome, v. 1, p. 1-376, 1992.

Stevens, J.D.; Bonfil, R.; Dulvy, N.K. \& Walker, P.A. The effects of fishing on sharks, rays and chimaeras (Chondichthyans), and the implications for marine ecosystems. ICES J. Mar. Sci., v. 57, p. 476-494, 2000.

Tiago, G.G.; Tutui, S.L.S.; Ávila-da-Silva, A.O.; Inácio, L.S.; Serrão, A.C.S; Koshikene, D. \& Santos, F.G. Pesca experimental e prospectiva com espinhel-de-fundo, in Anais da VIII Semana Nacional de Oceanografia, 107 p., Rio Grande-RS, 1995.

Tutuí, S.L.S. Comentários sobre a variação do coeficiente de capturabilidade em pescarias de espinhel de anzóis, Boletim do Instituto de Pesca, v. 26, n. 2, p. 203-210, 2000. 
Yulianto, I.; Booth, H.; Ningtias, P.; Kartawijaya, T.; Santos, J.; Kleinertz, S. \& Hammer, C. Practical measures for sustainable shark fisheries: Lessons learned from an Indonesian targeted shark fishery. Plos One, v. 13, n. 11, p. e0206437, 2018.

Wahrlich, R.; Perez, J.A.A.; Lopes, F.R.A. Aspectos tecnológicos da pesca do peixe-sapo (Lophius gastrophysus) com rede de emalhar no Sudeste e Sul do Brasil. Boletim do Instituto da Pesca, São Paulo, v. 30, n. 1, p. 87-98, 2004. 